\title{
Entropy and Equilibrium Property of QCD-Instanton Induced Final State in Deep-Inelastic Scattering*
}

\author{
Deng Weibing $^{1}$ Lu Ding $^{1}$ Zhang Jiying ${ }^{1}$ Xu Mingmei ${ }^{2 ; 1)} \quad$ Boris Levchenko $^{3}$ Liu Lianshou ${ }^{2}$ \\ 1 (College of Physical Science and Technology, Huazhong Normal University, Wuhan 430079, China) \\ 2 (Institute of Particle Physics, Huazhong Normal University, Wuhan 430079, China) \\ 3 (Skobeltsyn Institute of Nuclear Physics, Moscow State University, 119992 Moscow, Russian Federation)
}

\begin{abstract}
The scaling and additivity properties of Rényi entropy in rapidity space of the instanton final state (IFS) and current jet identified by the $r$-sorting method from the QCDINS Monte Carlo event sample are studied. Asymptotic scaling of Rényi entropy $H_{2}$ is observed for the IFS while the $\mathrm{H}_{2}$ for current jet tends to saturation with the decreasing of phase space scale. Furthermore, it is found that the additivity of $\mathrm{H}_{2}$ holds well for IFS in narrow rapidity window at different positions. These results indicate that the IFS produced in the instanton-induced process of deep inelastic scattering has reached local equilibrium.
\end{abstract}

Key words instanton; quark-gluon fusion; deep-inelastic scattering; entropy; local equilibrium

The basic theory of strong interaction — quan- 28 tum chromo-dynamics (QCD) is a non-Abelian gauge ${ }_{29}$ theory and as such has a complicated vacuum struc- 30 ture. There are degenerate vacua of different topolo- 31 gies, leading to a special effect — tunneling tran- 32 sition between degenerate vacua, referred to as instanton ${ }^{[1-4]}$. Instantons are non-perturbative fluctuations of the gluon field, which are expected to contribute into deep-inelastic scattering (DIS) with a sizable rate ${ }^{[5-7]}$.

Deep-inelastic scattering (DIS) accompanied by instanton-induced hard processes, can not be described by conventional perturbation theory. Therefore, theoretical and experimental studies of the pro- ${ }_{34}^{33}$ cesses induced by instanton are of fundamental sig- ${ }_{35}$ nificance for a thorough understanding of the nonperturbative sector of QCD ${ }^{[5,6]}$.

The two experimental groups, H1 and ZEUS, of DESY-HERA in Germany have been searching for ${ }^{38}$ instanton induced events in $\mathrm{e}^{+} \mathrm{p}$ deep inelastic scatter- ${ }^{39}$ ing experiments, and only upper limits on the crosssection of such processes were set ${ }^{[8,9]}$. At present, beside the experimental searching for the instanton induced events, a Monte Carlo study on the physical properties of IFS is also important.

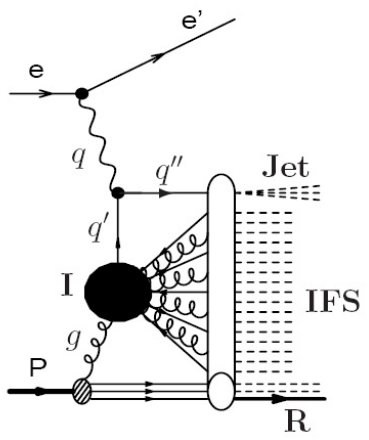

Fig1. The leading QCD-instanton induced process in the DIS regime of e-p scattering.

In figure 1 is shown the leading graph of QCDinstanton induced e-P collision. The incident lepton emits a photon, with 4-momentum $q$, which in turn transforms into a quark-antiquark pair. One of these quarks with 4-momentum $q^{\prime \prime}$ hadronizes to form the

Received xx February 200x

* Supported in part by the NSFC-RFBR cooperative research project 10475030 , NSFC project 10375025,90503001 and by the Ministry of Education of China NO CFKSTIP-704035.

1)E-mail:xumm@iopp.ccnu.edu.cn 
current jet. The other quark, with 4-momentum $q^{\prime},{ }_{85}$ fuses with a gluon (with 4-momentum $g$ ) from the 86 proton in the presence of an instanton (I). The hadron 87 system produced from the fusion of quark and gluon 88 in the presence of instanton is referred to as instanton 89 final state, or IFS in short.

The quark-gluon fusion process with the instanton 91 as background gives rise to a high multiplicity final 92 state. The particles produced from this process, i.e. 93 the IFS, are expected to be isotropically distributed 94 in their center of mass frame ${ }^{[10]}$.

A problem of high interest is whether the IFS 96 has reached equilibrium. Ref's. ${ }^{[11,12]}$ have studied 97 the final-state momentum distribution of IFS using 98 Monte Carlo simulation. An approximate isotropic distribution is found for the IFS in contrast to the high anisotropy of current jet. In the present letter the equilibrium of IFS will be studied by using the Rényi-entropy analysis based on Ma's coincidence ${ }^{100}$ probability.

Let us first discuss shortly the concept of equi- ${ }^{102}$ librium. For a closed macroscopic system, arriving at (thermal) equilibrium, the corresponding ensemble is the micro-canonical one, where the microscopic 103 states of the system are uniformly distributed in the 104 allowed region of phase space of the system ( $\Gamma$-space). Starting from this ensemble all the thermodynamical properties of the system, including the thermodynam- ${ }_{105}$ ical quantities — entropy, temperature, etc., are ${ }_{106}$ derived.

The physical origin of thermal equilibrium of a macroscopic system is the random collisions among ${ }_{109}$ the molecules constituting the system. In our case, each parton produced in the instanton-induced quarkgluon-fusion process develops a parton shower, re- ${ }_{112}$ spectively. Hadrons only experience resonance de- ${ }_{113}$ cay, if any. There is no rescattering, and no ran- ${ }_{114}$ dom thermal motion neither at parton level nor at ${ }_{115}$ hadron level. Therefore, no thermal equilibrium in the strict sense is possible. However, the distribution of the final states in the allowed region of phase space may be uniform. Such a distribution mimics the micro-canonical ensemble, and the "thermodynamical" properties of the system in consideration can be derived therefrom. We will refer to such a uniform distribution in phase space also as equilibrium. The "equilibrium", if any, of the IFS and current jet that we will check in the following should be understood in this sense.

To check the equilibrium of IFS and jet we will make use of the Rényi-entropy analysis based on Ma's coincidence probability ${ }^{[13-15]}$. According to $\mathrm{Ma}^{[16]}$ an event is characterized by a set of integer number $s \equiv\left\{m_{i}\right\}, i=1, \ldots, M$, where a certain phase space region $\Delta$ is partitioned equally into $M$ bins with the size of each bin $\delta=\frac{\Delta}{M}, m_{i}$ is the number of particles in the $i$ th bin. The number of coincidence of $k$ configurations is defined as

$$
N_{k}=\sum_{s} n_{s}\left(n_{s}-1\right) \cdots\left(n_{s}-k+1\right),
$$

where $n_{s}$ is the appearing time of the $s$-configuration in the whole event sample.

The coincidence probability of $k$ configurations is given by

$$
C_{k}=\frac{N_{k}}{N(N-1) \cdots(N-k+1)},
$$

where $N$ is the total number of events in the sample, and the Rényi entropies are defined as ${ }^{[17]}$

$$
H_{k} \equiv-\frac{\ln C_{k}}{k-1}
$$

The Rényi entropies are closely related to the Shannon entropy $(S)$, which is formally equal to the limit of $H_{k}$ as $k \rightarrow 1$ and can be obtained through extrapolation ${ }^{[14,15]}$. It has been shown that the extrapolation method for determining $S$ is in general not unique and will produce additional uncertainty ${ }^{[14,15]}$, while the Rényi entropies are of interest by themselves and can provide information on the equilibrium of the system, $c f$. Eq's. (5), (6) below. So our study will be concentrated on the behavior of Rényi entropies, in particular on $\mathrm{H}_{2}$.

For a system close to equilibrium the Rényi entropies obey the scaling property:

$$
H_{k}(M)=H_{k}\left(M_{0}\right)+d \ln \frac{M}{M_{0}}
$$

for sufficiently fine-grained subdivision of phase space ${ }^{[13,14]}$, where $d$ is a constant related to the di- 
mension of space, $M$ is bin number with the size of 156 each bin $\delta=\Delta / M, M_{0}$ is another bin number with ${ }_{157}$ the size of each bin $\delta_{0}=\Delta / M_{0}$. Substituting $M=\frac{\Delta}{\delta}, 158$ we can rewrite Eq. (4) as

$$
H_{k}(M)=A-d \ln \delta \text { for } M \rightarrow \infty,
$$

where $A=H_{k}\left(M_{0}\right)+d \ln \delta_{0}$ is a constant.

Furthermore, the additivity property ${ }^{[15]}$ :

$$
H_{k}^{(R)}(2 M)=H_{k}^{\left(R_{1}\right)}(M)+H_{k}^{\left(R_{2}\right)}(M),
$$
should hold for two non-overlapping and independent phase space regions $R_{1}$ and $R_{2}$ with $R$ the union. Note that the equality of the two sides of Eq. (6) is examined at the same scale $\delta=\frac{\Delta}{M}=\frac{2 \Delta}{2 M}$.

The above-mentioned method has been applied to hadron-hadron (h-h) collision data from both PYTHIA-JETSET Monte Carlo generator ${ }^{[18]}$ and ${ }^{13}$ NA22 experiment ${ }^{[19]}$. It turns out that in both cases the Rényi entropy $H_{2}$ versus $-\ln \delta y$ tends to saturate instead of approaching a straight line as expected by the scaling law Eq. (5) and the additivity Eq. (6) does not hold, showing that there is no equilibrium in the final state system of hadron-hadron collisions.

In the present letter we apply the method to the hadron system produced in the quark-gluon fusion process $q^{\prime}+g \stackrel{(\mathrm{I})}{\longrightarrow} X$ in the background of instanton. Our study is based on the Monte Carlo code QCDINS ${ }^{[20,21]}$, which is a package for instanton induced events embedded in the HERWIG ${ }^{[22,23]}$ event generator. The default parameters of the QCDINS 2.0 version are used in our study, i.e. $x^{\prime}>0.35$, $Q^{\prime 2}>113 \mathrm{GeV}^{2}$ and the number of quark flavors is set to be $n_{f}=3$.

In total 493400 instanton-induced DIS events are generated with the energies of electron and proton being equal to 27.5 and $820 \mathrm{GeV}$, respectively. The IFS and current jet are identified using the $r$-sorting ${ }_{183}$ method proposed in Ref. ${ }^{[11]}$. This method is to define a distance in the $\theta$ - $\phi$-plane in the hadronic center-ofmass frame, i.e.

$$
r(\theta, \phi)=\sqrt{\frac{\left(\frac{\theta-\theta_{0}}{\pi}\right)^{2}+\left(\frac{\phi-0}{\pi}\right)^{2}}{2},} \quad(7)^{185}
$$

where $\left(\theta_{0}, \phi_{0}=0\right)$ is the position of the current quark. This variable measures how far every final state particle is from the jet axis. Choosing an appropriate value for $r_{0}$ the particles with $r(\theta, \phi)<r_{0}$ are attributed to jet and those with $r(\theta, \phi)>r_{0}$ to IFS. First discard the proton remnant by an $1 \mathrm{D}$ cut. Renumber the left $n$ particles by their $r$ values, let $r_{1}<r_{2}<\cdots<r_{n}$. Choose the value of $r_{0}$ in between $r_{k}$ and $r_{k+1}\left(r_{k}<\right.$ $\left.r_{0}<r_{k+1}\right)$, i.e. take particles from 1 to $k$ as current jet, from $k+1$ to $n$ as IFS. Accumulating the energies from particle 1 to particle $k$ gives $E_{\mathrm{k}}=\sum_{i=1}^{k} \varepsilon_{i}$; simultaneously accumulating energies from $k+1$ to $n$ gives $E_{\mathrm{k}}^{\prime}=\sum_{i=k+1}^{n} \varepsilon_{i}$. The energy reconstruction errors for jet and instanton are $\Delta E_{\text {jet }}=\frac{E_{k}-E_{C}}{E_{C}} \times 100 \%$ and $\Delta E_{\mathrm{I}}=\frac{E_{k}^{\prime}-E_{I}}{E_{I}} \times 100 \%$, respectively, where $E_{C}$ is the energy of current parton, $E_{I}$ is that of the quark and gluon included in IFS at partonic level. Then choose the value of $k$ to optimize the energy reconstruction, i.e. make $\Delta E=0.4 \times\left|\Delta E_{\text {jet }}\right|+0.6 \times\left|\Delta E_{\mathrm{I}}\right|$ the minimum. The particles in the IFS and those in the current jet are then transformed to their own rest frames, respectively, for entropy analysis. The following referred rapidity $y$ are always in their rest frame. In Fig. 2 are shown the reconstruction error $\Delta E_{\text {jet }}$ for jet energy and $\Delta E_{\mathrm{I}}$ for instanton energy. It can be seen that the reconstruction is good and the resulting IFS and jet are reliable.
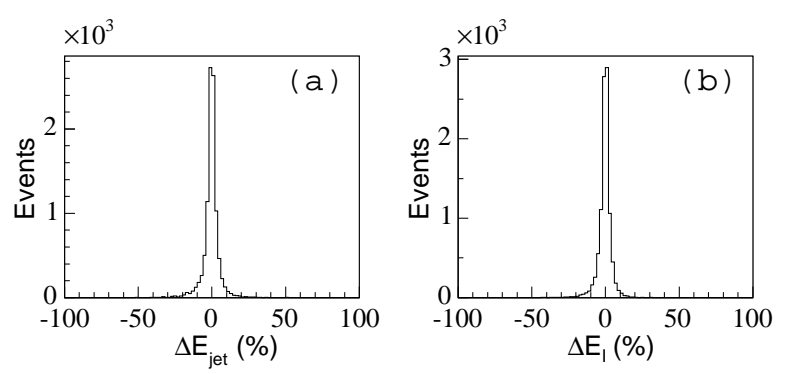

Fig2. The distributions of the reconstruction error $\Delta E_{\text {jet }}$ for jet energy (a) and $\Delta E_{\mathrm{I}}$ for instanton energy (b) in $r$-sorting method. 


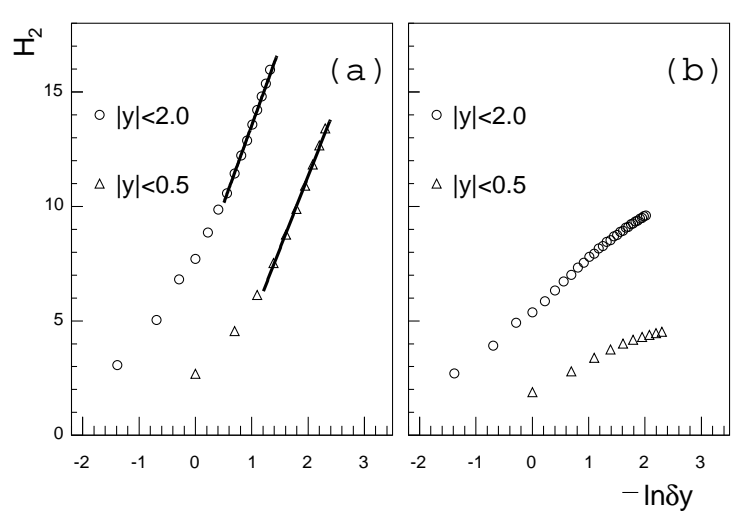

Fig3. The Rényi entropy $H_{2}$ versus $-\ln \delta y$ in central rapidity regions $|y|<2$ (circles) and $|y|<0.5$ (triangles) for (a) IFS and (b) current jet. The straight lines in (a) are linear fit to the high $-\ln \delta y$ part of $H_{2}$ as function of $-\ln \delta y$. The slopes of the two lines are 6.820 \pm 0.013 (upper line) and $6.304 \pm 0.005$ (lower line), respectively.

In Fig's. $3(a)$ and $(b)$ are shown the Rényi entropy ${ }_{231}$ $\mathrm{H}_{2}$ versus $-\ln \delta y$ in central rapidity regions $|y|<2_{232}$ (circles) and $|y|<0.5$ (triangles) for IFS and current jet, respectively. A striking feature is that, in ${ }_{234}$ case of IFS the behavior of $H_{2}$ versus $-\ln \delta y$ differs ${ }_{235}$ qualitatively from those for DIS-current jet and $h-h_{236}$ collision final states. It bends upwards and tends to $\mathrm{a}_{237}$ straight line for large $-\ln \delta y, c f$. Fig. $3(a)$, in contrast ${ }_{238}$ to the downward bending and saturation tendency for ${ }_{239}$ current jet (Fig 3(b)) and h-h collision final-state sys- ${ }_{240}$ tems ${ }^{[18,19]}$. This is a strong indication showing that ${ }_{241}$ unlike the violation of scaling law in cases of DIScurrent jet and h-h collision final-states the scaling ${ }_{243}$ law Eq. (5) is satisfied for IFS for sufficiently fine- ${ }_{244}$ grained subdivision of phase space.

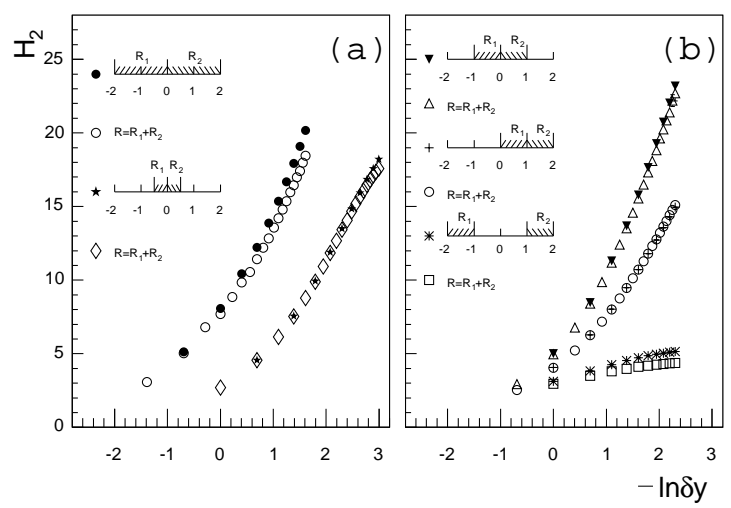

Fig4. The sum of Rényi entropy $H_{2}$ from IFS versus $-\ln \delta y$ for two regions $R_{1}$ and $R_{2}$ and
Fig the same width $(=1)$ but located at different places. It can be seen that when $R_{1}$ and $R_{2}$ are adjacent the additivity holds well, while when they are separated by a gap the additivity holds only approximately.

The asymptotic scaling and additivity of IFS are special and impressive. These properties have never been observed in other systems, e.g. hadronic jets and h-h collision final states, studied up to now. They strongly indicate that the system produced in quarkgluon fusion in the background of instanton, i.e. IFS, has reached local equilibrium.

Our Monte Carlo study is based on the QCDINS code, where the 4-momenta of the $n=2 n_{f}-1+n_{g}$ 246 247

that directly calculated from the whole region $R=R_{1}+R_{2}$. The legend shows the rapidity regions corresponding to each set of points.

Let us now check the additivity property Eq.(6) for the instanton final state IFS. In Fig. 4 are shown the Rényi entropies $\mathrm{H}_{2}$ of IFS obtained from taking the sum of the results calculated from two regions $R_{1}$ and $R_{2}$, in comparison with those directly calculated from the union $R=R_{1}+R_{2}$. The legend in the figure shows the rapidity regions corresponding to each set of points. For example, the solid circle in Fig. 4(a) is the sum of the $H_{2}$ 's from the regions $-2<y<0\left(R_{1}\right)$ and $0<y<2\left(R_{2}\right)$, while the open circle is the $H_{2}$ from the union $R=R_{1}+R_{2}(-2<y<2)$. It can be seen from Fig. 4(a) that when the widths of regions $R_{1}$ and $R_{2}$ are narrow $(\sim 0.5)$ the additivity holds excellently, while for wider widths $(\sim 2)$ the additivity holds only approximately, especially for large $M$. In produced partons in the instanton rest frame (for convenience, in the following we will refer to the partons produced in the instanton-induced quark-gluonfusion process as IQGF) are uniformly generated in energy-weighted phase space ${ }^{[20]}$, according to the leading-order matrix element ${ }^{[24]}$ with different energy weights for gluons and quarks. Every event of IQGF is a realization of the instanton-induced fusion process and can be regarded as a microscopic state of the system. The fact that the 4-momenta of the partons in IQGF are uniformly generated in energy-weighted phase space means that the micro- 
scopic states of IQGF are uniformly distributed in ${ }_{284}$ phase space, mimicking the micro-canonical ensem- 285 ble of a macroscopic system in thermal equilibrium. 286 In this sense the local equilibrium in parton level is ${ }_{287}$ built in the model due to theoretical consideration on 288 the property of instanton.

What we have checked above is the equilibrium 290 of the IFS, which is the hadron system after the 291 hadronization of IQGF. It is unclear whether and 292 how the equilibrium property of IQGF is preserved 293 in the hadronization process. Our work shows that 294 the local equilibrium is present in the IFS and can 295 be represented by the scaling and additivity of Rényi 296 entropy.

Since the IQGF and/or IFS are in local equilib- 298 rium we can derive the "thermodynamical" quantities 299 for them according to the usual thermodynamic for- 300 mulae. However, the meaning of the obtained values 301 of these quantities are different from those of a macro- 302 scopic system. For example, the temperature of a 303 macroscopic system measures the average kinetic en- 304 ergy of molecules, but the "temperature" in our case 305 do not have this kind of meaning. This explains why 306 the hadron system IFS can have temperature much higher than the critical temperature $(\sim 160-170 \mathrm{MeV})^{307}$ of deconfining phase transition, as shown in Ref. [11] ${ }^{308}$
In conclusion, we have studied the scaling and additivity properties of Rényi entropy $H_{2}$ for IFS and current jet in the instanton-induced process of deep inelastic scattering produced from the QCDINS Monte Carlo generator identified by the $r$-sorting method.

The Rényi entropy $H_{2}$ for IFS is found to obey the scaling law, Eq. (5), for sufficiently fine-grained phase space region and the additivity property, Eq. (6), holds in narrow phase space window at different positions, especially in adjacent intervals. These results provide strong indication that the IFS has reached local equilibrium.

In order to get a better understanding about the equilibrium of IFS, further investigation along this line is suggested, e.g. to study the entropy properties in 3 dimensional momentum space, transforming the variables to the corresponding cumulant ones ${ }^{[13]}$, or to use other characteristics of equilibrium, e.g. $\left|K_{l}(E, n)\right|^{2 / l} \ll K_{2}(E, n), l=3,4, \cdots,{ }^{[25-27]}$ which guarantees the smallness of the higher-order energy correlations $K_{l}(E, n)=\left\langle\prod_{k=1}^{l}\left(\epsilon_{k}-\langle\epsilon\rangle\right)\right\rangle$ with $\epsilon_{k}$ being the energy of the $k$ th particle.

The authors thank Li Zhiming for helpful discussions.

\section{References}

1 A. Belavin, A. Polyakov, A. Schwartz and Yu. Tyupkin, Phys. Lett. B 59, 85 (1975).

2 G. 't Hooft, Phys. Rev. Lett. 37, 8 (1976).

3 G. 't Hooft, Phys. Rev. D 14, 3432 (1976); Erratum in ${ }^{334}$ Phys. Rev. D 18, 2199 (1978).

4 G. 't Hooft, Phys. Rep. 142, 357 (1986).

5 S. Moch, A. Ringwald and F. Schrempp, Nucl. Phys. B ${ }^{337}$ 507, 134 (1997).

6 A. Ringwald and F. Schrempp, Phys. Lett. B 438, $217^{33}$ (1998).

7 A. Ringwald and F. Schrempp, Phys. Lett. B 459, $249^{341}$ (1999).

8 The H1 Coll., C. Adloff et al., Eur. Phys. J. C 25, 495-509 343 (2002).

The ZEUS Coll., S. Chekanov et al., Eur. Phys. J. C 34, 345 255-265 (2004).

10 A. Ringwald and F. Schrempp, Proc. 8th Int. Seminar, 347 Vladimir, Russia, 1994, D. Grigoriev et al. (eds.), p. 170. 348 World Scientic, Singapore (1995) [hep-ph/9411217].

$11 \mathrm{Xu}$ Mingmei et al., in preparation.
12 Zhang Kunshi et al., in preparation.

13 A. Białas, W. Czyż and J. Wosiek, Acta Phys. Pol. B 30 107 (1999).

14 A. Białas and W. Czyż, Phys. Rev. D 61, 074021 (2000).

15 A. Białas and W. Czyż, Acta Phys. Pol. B 31, 687 (2000).

16 S. K. Ma, Statistical Mechanics (World Scientific, Singapore, 1985), p. 425; S. K. Ma, J. Stat. Phys. 26, 221 (1981); S. K. Ma and M. Payne, Phys. Rev. B24, 3984 (1981).

17 A. Rényi, Acta Math. Acad. Sci. Hung. 10, 193 (1959).

18 K. Fialkowski and R. Wit, Phys. Rev. D 62, 114016 (2000).

19 NA22 Coll., M. R. Atayan et al., Acta Phys. Pol. B 36, 2969 (2005).

20 A. Ringwald and F. Schrempp, Comput. Phys. Commun. 132, 267 (2000) [hep-ph/9911516].

21 M. Gibbs, A. Ringwald and F. Schrempp, in: Proc. DIS 1995 (Paris, France, 1995), J.-F. Laporte, Y. Sirois, Eds., pp. 341, hep-ph/9506392.

22 G. Marchesini and B. Webber, Nucl. Phys. B 310, 461 (1988).

23 G. Marchesini et al., Comput. Phys. Commun. 67, 465 (1992). 
35124 S. Moch, A. Ringwald and F. Schrempp, Nucl. Phys. B 354 507, 134 (1997)

35325 J. Manjavidze, A. Sissakian, hep-ph/0204281.
26 A. N. Sissakian, Physics Uspekhi 46, 3 (2003), or download at http://www.ufn.ru/archive/Index03.html\#rel_2_2003_303.

35627 J. Manjavidze, A. Sissakian, Phys. Rept. 346, 1 (2001). 


\section{深度非弹性散射中瞬子末态的熵分析及热平衡性质*}

1 (华中师范大学物理科学与技术学院理科基地班2004级＼cjkstart武汉４30079) 2 (华中师范大学粒子物理研究所 武汉 430079)

3 (莫斯科大学斯科别尔琴核物理研究所 莫斯科 119992 俄罗斯)

摘要 在QCDINS 产生的瞬子参与的ep深度非弹性散射事件中用 $\mathrm{r}$ 排序法得到的瞬子末态和喷注样本, 研究 了Renyi 熵在快度空间中的标度性和相加性。在瞬子未态中观察到了Renyi 摘随着相空间尺度的减小展现出来 的渐进标度性, 而喷注展现出饱和趋势。在瞬子末态中还观察到在不同快度位置的窄窗口中Renyi 熵的相加性 是很好地成立的。这些现象表明在瞬子参与的深度非弹性散射子中的瞬子末态达到了局域热平衡。

关键词 瞬子 夸克胶子融和 深度非弹性散射摘分析 热平衡性质

*中国国家自然科学基金一俄罗斯基础研究基金合作研究项目10475030，国家自然科学基金10375025，90503001，国家教委重大项目 培育基金704035资助

1)E-mail:xumm@iopp.ccnu.edu.cn 